\title{
Regulation of c-ski Transgene Expression in Developing and Mature Mice
}

\author{
John M. Leferovich, ${ }^{1, a}$ Dolores P. Lana, ${ }^{2, a}$ Pramod Sutrave, ${ }^{2, a}$ Stephen H. Hughes, ${ }^{2}$ and Alan M. Kelly' \\ 'Department of Pathobiology, School of Veterinary Medicine, University of Pennsylvania, Philadelphia, Pennsylvania \\ 19104, and ${ }^{2}$ ABL-Basic Research Program, N.C.I-Frederick Cancer Research and Development Center, Frederick, \\ Maryland 21702-1201
}

\begin{abstract}
The control of c-ski transgene expression and muscle hypertrophy have been investigated in transgenic mice. In adult animals, the level of transgene expression is linked to the specialized phenotype of individual muscles, high levels occur in fast muscles and significantly lower levels in muscles with high metabolic activity (diaphragm, soleus). These findings have led us to propose that a threshold must be passed before ski-induced growth can occur. We now show that within fast muscles, induced hypertrophy uniquely involves Illb fibers. This pattern of expression is under development control; levels of c-ski mRNA are low in all muscles at birth. In the diaphragm, there is a sevenfold increase in c-ski message levels between $5 \mathrm{~d}$ and maturity. By contrast, in fast extensor digitorum longus and anterior tibial muscles, there is a 24-fold increase in levels between 5 and $12 \mathrm{~d}$ postpartum. Muscle hypertrophy and antibody staining for c-skiprotein in myofiber nuclei emerge concurrently. This pattern of c-skiexpression parallels the appearance of Ilb myosin heavy chain transcripts (Wydert et al., 1987) and differentiation of Ilb fibers, suggesting that amplification of c-skimRNA levels is linked to the development of Ilb fiber specialization. Manipulations that are known to perturb Ilb fiber development, neonatal denervation, and neonatally induced hypothyroidism inhibit high levels of c-ski expression and hypertrophy. In the adult fast EDL, denervation leads to rapid atrophy of Ilb fibers and a significant decline in levels of c-ski mRNA. The results suggest that the environment of differentiated Ilb fibers permits the expression of high levels of c-sk/mRNA and this, in turn, induces hypertrophy.
\end{abstract}

[Key words; c-ski, transgene, development, hypertrophy, control, neural]

The role of endogenous c-ski in normal animals is unknown; it is expressed at low levels in many tissues throughout development (Stavnezer, 1988). We have shown that alternative

\footnotetext{
Received Dec. 30, 1993; revised July 6, 1994; accepted July 6, 1994.

We wish to thank Drs. Robin Fitzsimons, Stefano Schiaffino, and Edward Stavnezer for their gifts of monoclonal antibodies that made this study possible. We are equally indebted to Drs. Margaret Buckingham and Christos Petropoulos for their gifts of cDNA probes. To Ms. Kathy Haeber, we extend our thanks for her expert technical assistance in constructing illustrations. These studies were supported in part by grants from the USPHS (HL 15835 to the Pennsylvania Muscle Institute), from the Muscular Dystrophy Association of America and from the National Cancer Institute, DHHS, under Contract NO1-CO-74101 with ABL.

Correspondence should be addressed to Alan M. Kelly at the above address.

a.M.L., D.P.L., and P.S. contributed equally to the reported research.

Copyright (C) 1995 Society for Neuroscience $0270-6474 / 95 / 150596-08 \$ 05.00 / 0$
}

splicing of c-ski RNA generates at least three messages, and by implication, three different protein isoforms (Sutrave and Hughes, 1989). Available evidence however, suggests that none of these isoforms is muscle specific (Sutrave and Hughes, 1991).

When cultured avian cells are infected with viruses that express high levels of $\mathrm{v}$-ski or c-ski, two dissimilar effects are seen; (1) chick embryo fibroblasts undergo morphological transformation, proliferate, and grow in soft agar (Stavnezer et al., 1981, 1986; Li et al., 1986), and (2) quail body wall cells are induced to terminally differentiate into striated muscle (Colmenares and Stavnezer, 1989; Colmenares et al., 1991). These findings present an apparent paradox since most current dogma holds that cell proliferation and differentiation are mutually exclusive cvents. Wc asked if c-ski would producc cither of these effects when expressed at high levels in transgenic animals. Several independent lines of transgenic mice were created in which a truncated c-ski cDNA was placed under the transcriptional control of the Moloney murine sarcoma virus (MSV) long terminal repeat (LTR) (Sutrave et al., 1990b, 1992).

In three lines of transgenic mice we found remarkable hypertrophy of almost every skeletal muscle in the body (Sutrave et al., 1990b). One of these lines, 8566, was examined in detail. Most of the skeletal muscles of this line contain levels of transgene RNA many hundred-fold higher than the levels of mRNA from the endogenous c-ski gene. Most fast muscles in this transgenic line were significantly increased in size. Preliminary evidence indicated that this is due to a selective hypertrophy of type IIb and possibly IIx fibers; types I and IIa fibers of these same muscles do not appear to be enlarged. Apart from hypertrophy, both the muscle architecture and numbers of myonuclei are normal. These observations imply that high level expression of c-ski induces hypertrophy without initiating either satellite cell proliferation and or fusion into the growing fibers.

The soleus and diaphragm are exceptions to the generalized hypertrophic response. Fibers of these muscles are not enlarged; coupled with this is the finding that the levels of mRNA from the c-ski transgene are significantly lower than levels in hypertrophied muscles. These observations suggest that there is a threshold for induction of the ski phenotype. Since the soleus and diaphragm are muscles that sustain high functional demands, the results also suggest that neurally imposed activity may play a role in regulating ski expression.

These findings presented an opportunity to analyze the control of $s k i$ transgene expression and of the hypertrophy it causes. The transgene is under the transcriptional control of the MSV promotor and in the transgenic animals, the transcripts are appropriately initiated within the MSV LTR (Sutrave et al., 1990b). 
When linked to other genes, the MSV LTR is expressed in a variety of tissues in transgenic mice (Khillan et al., 1987). By contrast, when the MSV LTR is linked to c-ski in transgenic mice, expression is limited to skeletal muscle, and the level of expression depends on the specialized phenotype of individual muscles. Since muscle hypertrophy has developed in three lines of MSV-ski transgenic mice (Sutrave et al., 1990) the reaction is not integration site specific. Nor is it species specific for a similar hypertrophic response has arisen in MSV-ski transgenic pigs (Hughes et al., in preparation).

In the present study we have followed the development of the ski phenotype in developing muscle and have investigated the effects of thyroid hormone and of neural stimulation on expression of the transgene. We show that levels of mRNA from the c-ski transgene are low in all muscles at birth and increase with age. This increase involves all muscles, both fast and slow. However, muscles with high functional demands always have the lowest basal levels of c-ski mRNA from the transgene. Within fast muscles, c-ski mRNA and protein accumulates to high levels in parallel with the differentiation of IIb fibers. Neural or hormonal perturbation of IIb fiber differentiation, inhibit accumulation of high levels of c-ski mRNA. The results suggest that the environment of the IIb phenotype permits the expression of high levels of $\mathrm{c}-s k i$ mRNA and protein.

\section{Materials and Methods}

Animals. Mice of line 8566 are heterozygotes. Animals that carry the c-ski transgene were identified by dot blot analysis of DNA extracted from tail clips (data not shown).

Tissue preparation. Individual muscles as well as whole hind limbs were frozen in isopentane cooled to $-160^{\circ} \mathrm{C}$ in liquid nitrogen. For immunochemistry and fiber morphometry, the entire length of individual muscles was affixed at rest length to a length of polystyrene and frozen. The frozen muscle was then cut precisely in the midbelly with a razor and reoriented for sectioning in a transverse plane. Muscles used in molecular procedures were frozen directly in liquid nitrogen. All tissues were stored at $-120^{\circ} \mathrm{C}$ prior to histochemical and molecular analysis. Immunohistochemistry and morphometry were performed on 8-10 micron-thick frozen sections cut through the midbelly of each muscle.

Immunocytochemistry. Fiber types were identified by indirect immunofluorcscence staining using monoclonal antibodies specific for the slow myosin heavy chain (MHC) (NOQ7.5 4D, Narusawa et al., 1987), for 2a MHC (SC71), 2b MHC(BF-F3), and specific for all of the above myosin types except 2 X (BF-35, Schiaffino et al., 1989). Staining protocols have been previously described (Narusawa et al., 1987). Fibertype proportions were determined by fiber counts made on serial sections, each stained with a specific anti-myosin antibody. Sections were photographed and fiber numbers tabulated from prints assembled as a collage.

For detection of ski protein, sections were fixed in 3\% formalin for 10 min, rinsed four times in PBS, incubated in $0.1 \%$ Triton-X in PBS for $10 \mathrm{~min}$, then rinsed in PBS. Following a $20 \mathrm{~min}$ incubation in 3\% normal goat serum in PBS, sections were incubated for $60 \mathrm{~min}$ with anti-ski monoclonal antibody, G-8 (gift from Dr. Ed Stavnezer, see Sutrave et al., 1990a), dilutcd 1:50 with PBS. After washing with PBS, sections were incubated for $20 \mathrm{~min}$ with fluorescein conjugated goat anti-mouse I-gG (Cappel) secondary antibody diluted 1:500 in PBS.

Morphometry. Fiber sizes were determined from photographic enlargements of muscle sections stained with hematoxylin and eosin. Fibers were measured using SIGMASCAN software in combination with a digitizing tablet (Jandel Scientific).

Normalized weight. Measurements of muscle weight-to-body-weight ratios were made using the wet weight of muscles frozen in liquid nitrogen immediately after removal.

Hypothyroidism. Animals were given $0.05 \%$ phenylthiouracil in their drinking water according to previous methods (Gambke et al., 1983).

Denervation. All surgeries were performed using Halothane anesthesia. In young and adult mice, denervation of the left hind limb was
Table 1. Comparison of the wet weights of selected muscles from control and c-ski transgenic mice

\begin{tabular}{lrcc} 
& Control mice & $\begin{array}{l}\text { Transgenic } \\
\text { (line 8566) }\end{array}$ & \% Change \\
\hline Vastus lateralis & $4.43 \pm 0.71$ & $7.07 \pm 1.04$ & $+62 \%$ \\
Triceps & $2.67 \pm 0.32$ & $3.55 \pm 0.22$ & $+33 \%$ \\
Semitendinosus & $1.61 \pm 0.43$ & $2.94 \pm 0.71$ & $+83 \%$ \\
Vastus intermedius & $0.33 \pm 0.01$ & $0.36 \pm 0.12$ & $+9 \%$ \\
Aductor longus & $0.26 \pm 0.17$ & $0.19 \pm 0.105$ & $-27 \%$ \\
Sternohyoidius & $0.50 \pm 0.15$ & $1.18 \pm 0.27$ & $+134 \%$ \\
Sternomastoideus & $0.59+0.02$ & $1.08 \pm 0.25$ & $+83 \%$ \\
Anterior tibialis & $1.38 \pm 0.10$ & $2.80 \pm 0.2$ & $+102 \%$ \\
Extensor digitorum & & & \\
$\quad$ longus & $0.335 \pm 0.33$ & $0.72 \pm 0.14$ & $+114 \%$ \\
Gastrocnemius & $3.56 \pm 0.56$ & $5.03 \pm 0.48$ & $+41 \%$ \\
Plantaris & $0.52 \pm 0.06$ & $0.765 \pm 0.11$ & $+47 \%$ \\
Soleus & $0.305 \pm 0.04$ & $0.253 \pm 0.14$ & $-17 \%$ \\
Diaphragm & $3.97 \pm 0.29$ & $3.71 \pm 0.22$ & $-5 \%$ \\
\hline
\end{tabular}

Measurements were made from three control and three transgenic mice. Since these animals were not matched for size, the results are expressed as wet weight normalized to total body weight.

accomplished by surgical removal of a 1 to $2 \mathrm{~mm}$ segment of the sciatic nerve in the proximal thigh.

$R N A$ analysis. Total RNA isolated from the muscles of the hind limb was frozen in liquid nitrogen immediately following dissection, homogenized in RNAzol (Tel-Test), and processed according to the manufacturer's protocol.

RNase protection assays were carried out essentially as described (Sutrave et al., 1990b). A 420 base pair (bp) EcoRI-BAMHI c-ski fragment was subcloned in the Bluescript $\mathrm{KS}$ vector (Stratagene) and used to generate radioactively labeled antisense RNA from the $T_{7}$ promoter. The actin probe was generated in the same manner using a $240 \mathrm{bp}$ fragment from the $3^{\prime}$ noncoding region of the $\alpha_{\mathrm{sk}}$ actin gene (gift from Dr. C. J. Petropoulos). A combination $s k i$-actin probe $(671 \mathrm{bp})$ in which the actin fragment served as an internal standard, was used to generate the RNase protection assay shown in Figure 6.

The procedure for Northern transfer analyses was as previously described (Sutrave et al., 1990a). The probe for Northern transfer analysis of IIb MHC mRNA was a DNA fragment corresponding to the $3^{\prime}$ untranslated region of the mRNA (Lyons et al., 1990, a gift from Dr. M. Buckingham).

\section{Results and Discussion}

Sizes of adult muscle

We have analyzed the effects of c-ski on a variety of muscles in order to understand the regulation of transgene expression more fully. A selected group of 13 muscles were carefully dissected from control and line 8566 transgenic animals and the wet weights recorded (Table 1). Since we were unable to match the control and transgenic animals for size, the results of the analysis are expressed as mean weights normalized to total body weight. Among the 13 muscles, there is considerable variation in the percentage increase of normalized weight in line 8566 mice relative to controls. The greatest increase is in the sternohyoideus, which is expanded by $134 \%$ compared to control. By contrast, the diaphragm and vastus intermedius have essentially the same weight as in controls, and the soleus and adductor longus muscles of 8566 mice are decreased in relative weight by $17 \%$ and $27 \%$, respectively. For both the soleus and adductor longus, this decline represents an absolute decrease in the weight of the muscle. When not corrected for body weight, for example, the mean weight of the soleus declined from $8.2 \pm 0.5 \mathrm{mg}$ in 


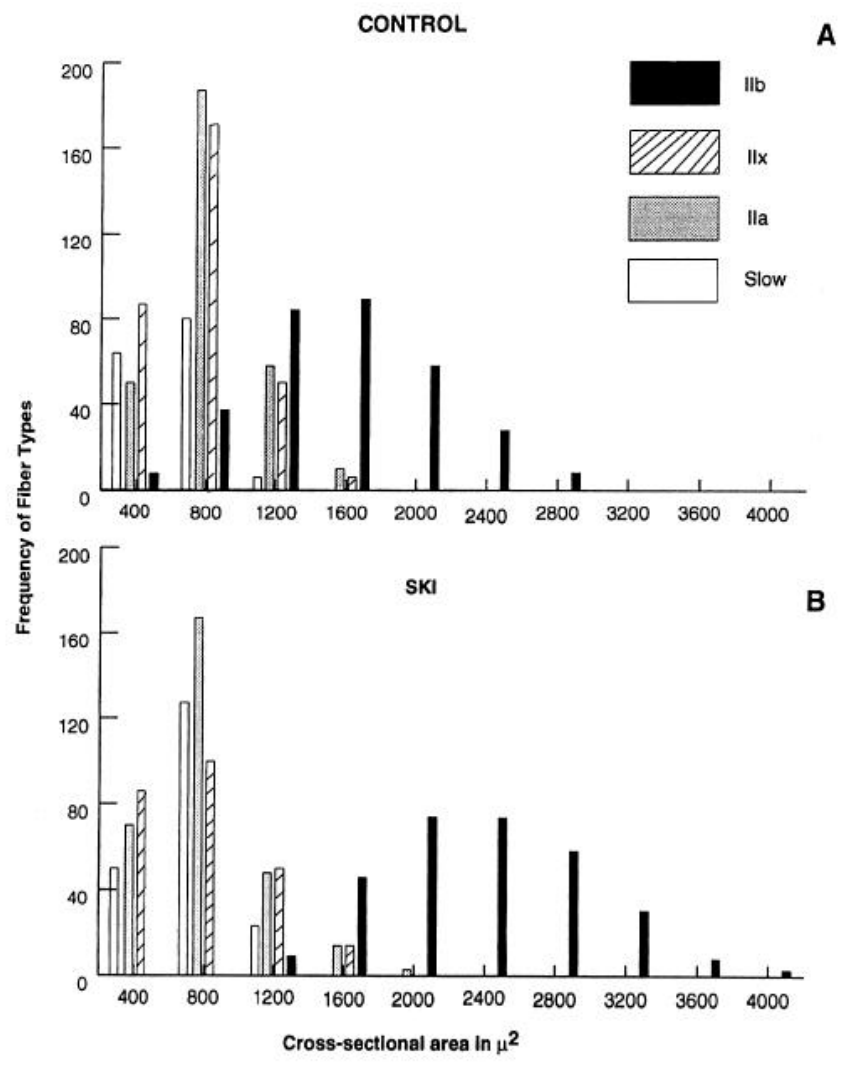

Figure 1. Comparison of the cross-sectional areas of specialized fiber types in the mature EDL of control and line $8566 \mathrm{c}$-ski transgenic mice. Transverse cryostat sections taken through the middle of the muscle belly were stained with monoclonal antibodies specific to each of the fiber types. There is no difference in the size of slow (type I), IIa or IIx fibers between the two groups of animals; hypertrophy uniquely involves type IIb fibers in c-ski mice.

control to $6.6 \pm 0.7 \mathrm{mg}$ in transgenic mice. The explanation for the diminished growth is presently unavailable.

In the lower hind limb, muscles of the anterior compartment, the anterior tibial and EDL, hypertrophy to a significantly greater extent than the gastrocnemius and plantaris in the posterior compartment. The explanation for this difference is not clear but may be correlated with the fact that muscles of the posterior compartment play a greater role in postural activity.

\section{Fiber specialization}

In previous studies, we showed that Types I and IIa fibers were not hypertrophied in c-ski transgenic mice. At that time, we could not clearly differentiate IIx from IIb fibers. We have now reexamined this question using a panel of monoclonal antibodies (kindly made available by Dr. Stephano Schiaffino, see Schiaffino et al., 1989) that distinguish Types IIx and IIb fibers in addition to types I and IIa fibers. Muscles were sectioned transversely through the midbelly and cross-sectional areas of specific fiber types were measured using a digitizer. The results (Fig. 1), show that the cross-dimensions of types I, IIa, and IIx fibers in c-ski mice are not significantly different from controls, and that hypertrophy is due exclusively to growth of type IIb fibers.

To determine if expression of c-ski induces a redistribution in the proportions of fiber types in muscle, serial transverse sections were taken from the midbelly of the EDL, plantaris, and diaphragm of three control and three line 8566 mice and were stained with the panel of antibodies. Sections were photographed so that the entire cross section of the muscles could be assembled as a montage, and the frequency of each fiber type was analyzed in control and line 8566 muscles mice (Table 2).

In the EDL and plantaris of the ski transgenic mice there was a shift in phenotype from IIx to IIb. The greatest shift is in the plantaris in which there is a $14 \%$ increase in proportions of IIb fibers. This increase is paralleled by a decline in the proportions of IIx, IIa, and slow fibers. The increase in proportion of IIb fibers in the EDL is smaller than in the plantaris and appears to be due, primarily, to a switch in fibers from the IIx to the IIb phenotype; there is little change in the proportions of IIa or slow fibers in this muscle.

These results show that c-ski specifically augments the growth of IIb fibers and has the capacity to override the controls regulating patterns of muscle fiber specialization. The transition from the IIx to IIb phenotypes may be partially accounted for by the observation that in controls, a small number of fibers that were defined as type IIx also contained low levels of IIb MHC. In the transgenic mice, the levels of IIb myosin in these fibers may have been amplified so that they were identified as IIb.

Using myosin ATPase staining, the proportion of slow fibers was measured in several of the muscles identified in Table 1. The soleus, adductor longus, and vastus intermedius and diaphragm, respectively, contain $47 \%, 48 \%, 59 \%$, and $23 \%$ type I fibers. These muscles contain no IIb fibers. Hence, muscles that

Table 2. Frequency of fiber type

\begin{tabular}{|c|c|c|c|c|}
\hline Muscle & IIb & IIx & $\mathrm{IIa}$ & I \\
\hline \multicolumn{5}{|l|}{ EDL } \\
\hline Control & $44.7 \pm 4.8$ & $22.4 \pm 3.2$ & $23.3 \pm 1.04$ & $9.5 \pm 2.6$ \\
\hline 8566 & $53.2 \pm 4.8$ & $13.4 \pm 0.06$ & $24.8 \pm 4.6$ & $8.5 \pm 2.7$ \\
\hline \multicolumn{5}{|l|}{ Plantaris } \\
\hline Control & $58.2 \pm 8.4$ & $16.5 \pm 3.0$ & $20.5 \pm 5.6$ & $4.7 \pm 0.36$ \\
\hline 8566 & $72.2 \pm 2.7$ & $10.1 \pm 1.1$ & $15.5 \pm 3.06$ & $2.2 \pm 1.2$ \\
\hline \multicolumn{5}{|l|}{ Diaphragm } \\
\hline Control & & 35.9 & 56.5 & 8.9 \\
\hline 8566 & & 33.8 & 58.9 & 7.3 \\
\hline
\end{tabular}



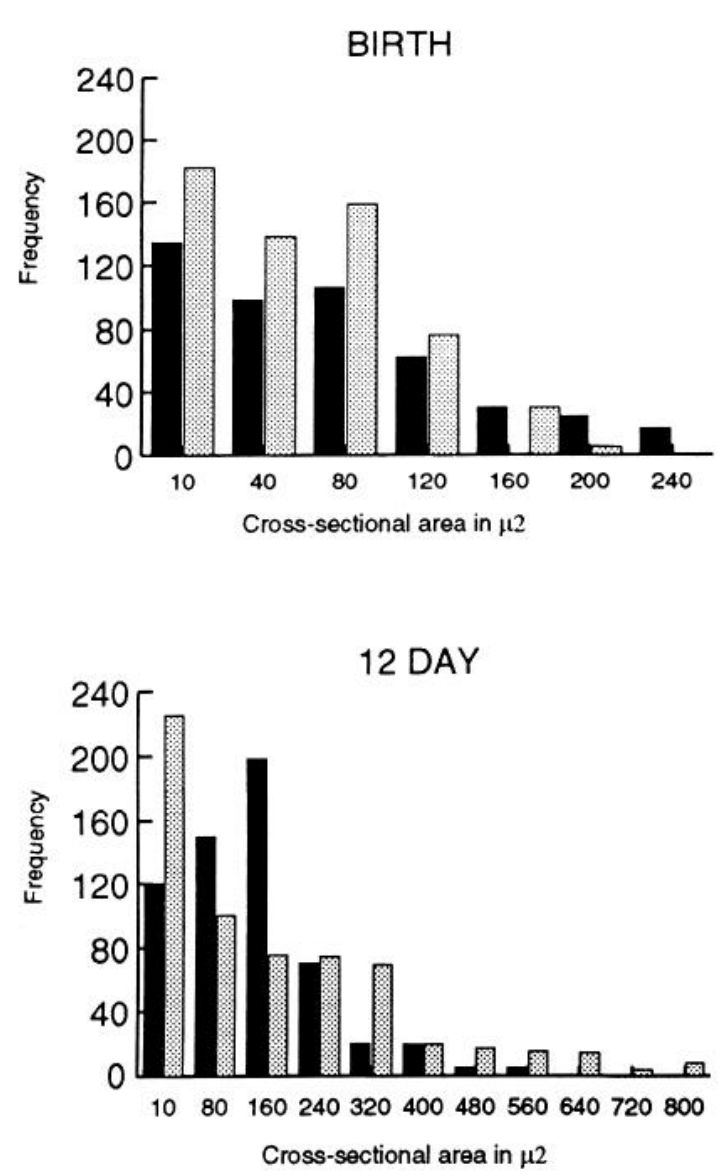
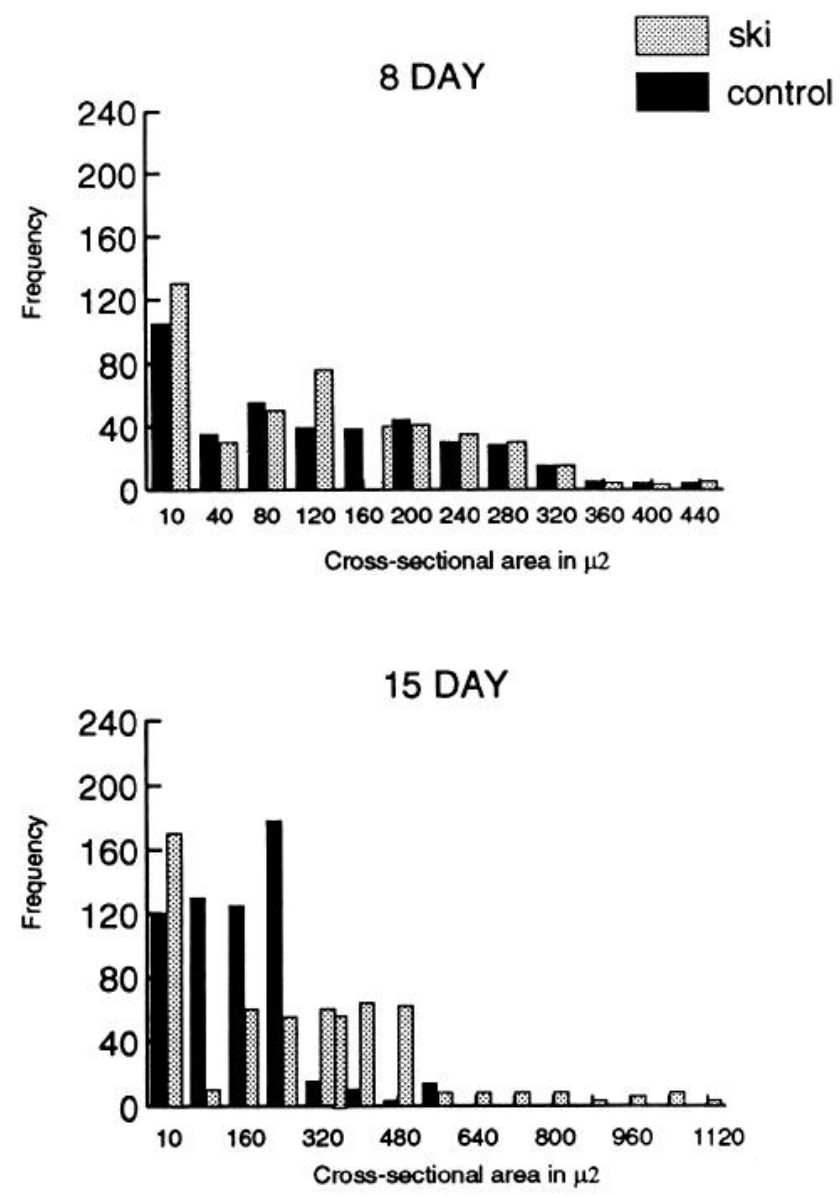

Figure 2. Development of the ski-induced phenotype. Fiber cross-sectional areas were measured on $\mathrm{H}$ and $\mathrm{E}$ stained, transverse cryostat sections made through the middle of the middle of the EDL and anterior tibial muscles of control and transgenic mice at successive stages of development. There is no difference in fiber size between $s k i$ and control animals between birth and $8 \mathrm{~d}$. Hypertrophy emerges in developing c-ski mice between 8 and $12 \mathrm{~d}$. This response is amplified by $15 \mathrm{~d}$.

contain large numbers of slow fibers and no IIb fibers do not increase in wet weight. By contrast, the EDL, plantaris, anterior tibialis, and sternohyoideus muscles contain comparatively few slow fibers (respectively, $9.5 \%, 4.7 \%, 2 \%$, and $0 \%$ ), and large numbers of type IIb fibers. These are muscles that significantly increase in wet weight. $S k i$-induced hypertrophy can, therefore, be directly linked to specialization of the IIb phenotype. However, other, local factors must be involved in regulating hypertrophy since the EDL and plantaris (respectively, anterior and posterior compartment muscles) contain approximately the same proportions of IIb fibers yet the EDL is increased in wet weight by $114 \%$ and the plantaris by only $47 \%$.

\section{Expression of myosin heavy chains}

Levels of IIb MHC mRNA in skeletal muscle from mature control and c-ski mice were analyzed on Northern blots using a probe that is specific to the $3^{\prime}$ UTR of the IIb MHC transcript (Lyons et al., 1990, gift from Dr. M. Buckingham). Densitometric scans of the blots show there is approximately a twofold increase in IIb MHC mRNA levels in anterior tibial and EDL muscles from the transgenic mice compared to controls (data not shown).

\section{Development of the c-ski-induced phenotype}

Extensor digitorum longus (EDL) muscle was obtained from control and transgenic mice at successive stages of development form birth to maturity. Transverse, cryostat sections were obtained from the middle of the muscle belly and the entire cross section of the muscle was stained and photographed. Fiber crosssectional areas were measured from these photos using a computer-based image processing system (sIGMASCAN, Jandel Scientific).

In the developing EDL, there is no difference in fiber size between 8566 and control mice from birth to $8 \mathrm{~d}$ postpartum (Fig. 2). In the control EDL at $12 \mathrm{~d}$, the largest fibers have a cross-sectional area of $560 \mu \mathrm{m}^{2}$. Between 8 and $12 \mathrm{~d}$, the size of some of the fibers in ski mice increases significantly with the result that at $12 \mathrm{~d}$ the largest fibers have cross-sectional areas of $800 \mu \mathrm{m}^{2}$; nearly $40 \%$ greater than the equivalent controls. This difference in dimension increases as muscles of 8566 mice advance to maturity (Fig. 2).

These results show that emergence of the c-ski phenotype is stage specific and dependent upon the state of muscle differentiation. The response appears comparatively late in development at a time when the number of fibers in a muscle is already determined and hypertrophy is probably the only option available to growth stimulation.

\section{Expression of c-ski mRNA in developing muscle}

Total RNA was isolated from the anterior tibial muscle of transgenic mice at birth, $5,8,12$, and $15 \mathrm{~d}$ postpartum and in the adult. Transcripts that derive from the c-ski transgene were 
A

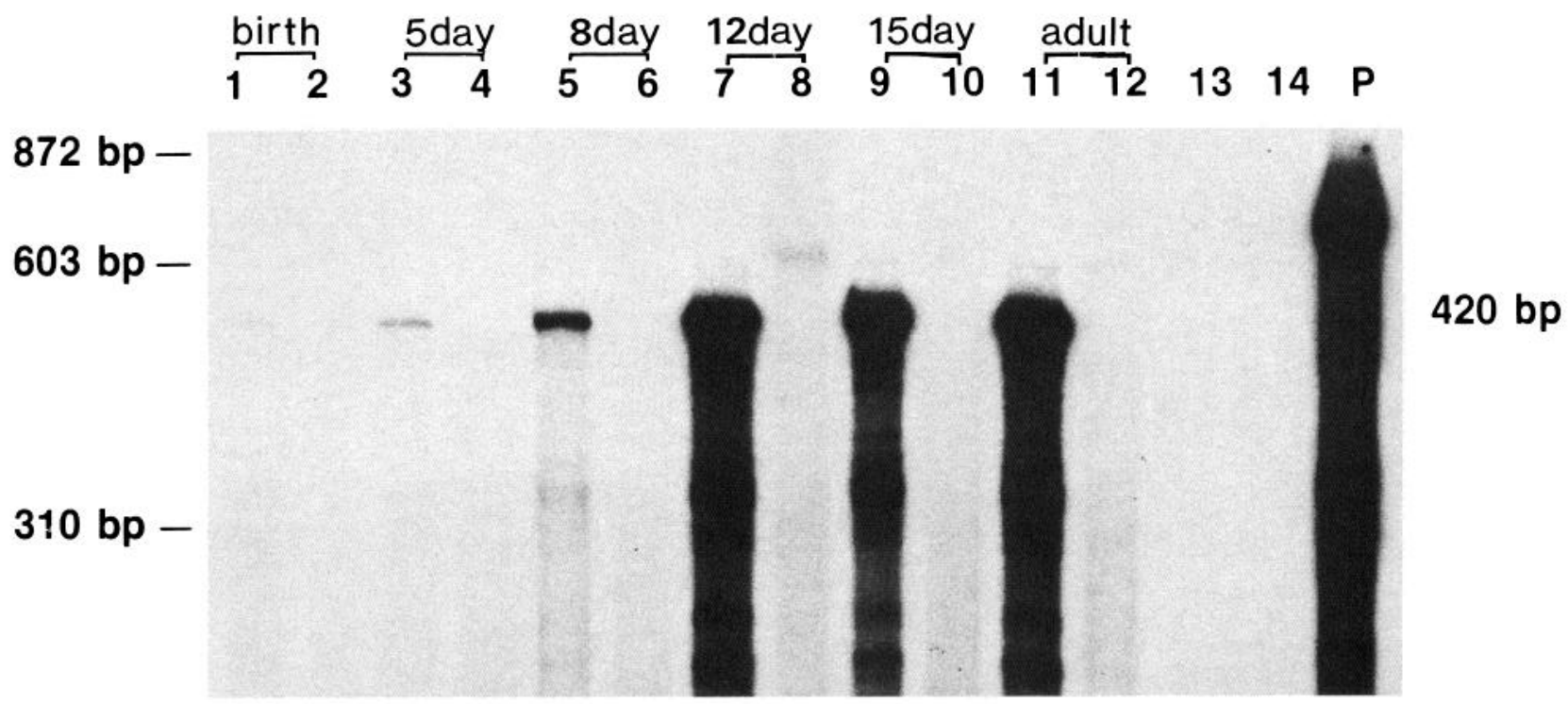

B

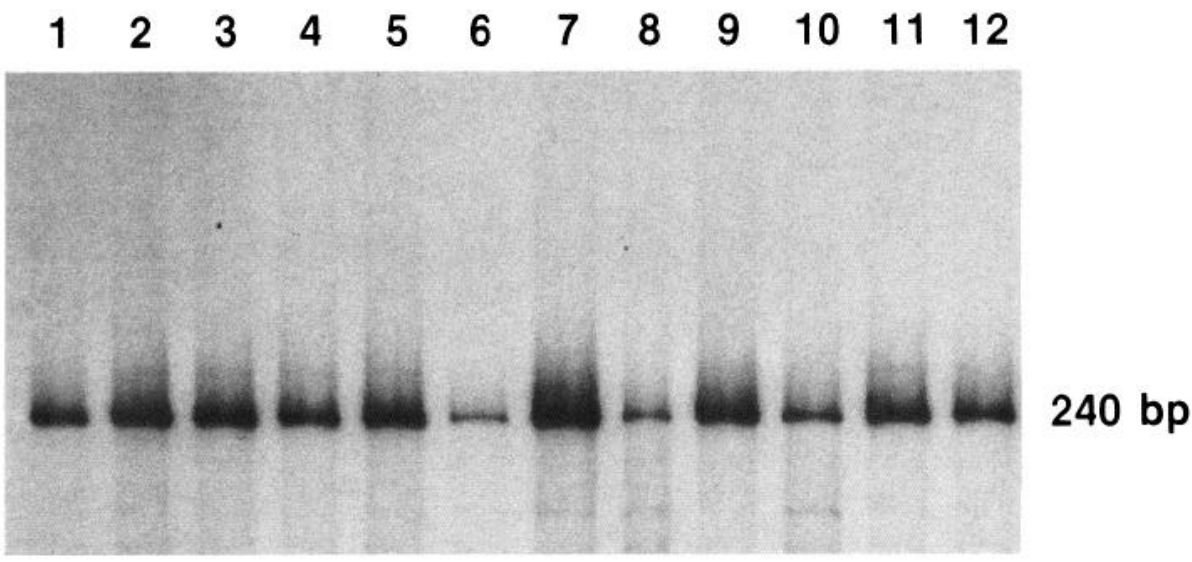

Figure 3. Pattern of c-ski mRNA expression in the developing Anterior tibial muscle. A, RNase protection assay demonstrating temporal expression of c-ski mRNA in the anterior tibialis muscle of transgenic mice. A 420-base uniformly labeled antisense probe (lane P) was hybridized to $15 \mu \mathrm{g}$ of total RNA. RNase-resistant fragments from digestion of the RNA-RNA hybrids made using RNA from the anterior tibialis muscle are shown in lanes 1-12. Lanes 1, 3, 5, 7, 9, and 11 are from leg muscle of ski transgenic mice at birth, 5, 8, 12, and $15 \mathrm{~d}$ of development and in the adult. Lanes 2, 4, 6, 8, 10, and 12 are from age-matched control animals. Lane 13 shows tRNA control and lane 14 is the probe control. Numbers to the left are sizes in bases as indicated by a PhiX 174 Hae III digested DNA marker. Number to the right indicate the size of the RNase-resistant fragments. Levels of c-ski mRNA are low at birth and $5 \mathrm{~d}$. By $12 \mathrm{~d}$, transcript levels approximate those of the adult. $B$, RNase protection assay demonstrating the expression of endogenous $\alpha_{\mathrm{sk}}$ actin. A 240-base uniformly labeled antisense probe was hybridized to $15 \mu \mathrm{g}$ of total RNA. RNaseresistant fragments from digestion following hybridizations with RNA from the anterior tibialis muscle are shown in lanes $1-12$ and correspond to the samples indicated in $A$. Numbers to the right indicate the size of the RNase-resistant fragments.

analyzed by RNAse protection assay using probes that protect a 420 bp fragment of c-ski mRNA and as reference, a $240 \mathrm{bp}$ fragment of skeletal muscle $\alpha$-actin mRNA.

We have previously shown that levels of transgene mRNA in the mature diaphragm are significantly lower than in other fast muscles (Sutrave et al., 1990b, see also Fig. 4). At 5 d, the level of c-ski mRNA in the developing diaphragm is notably lower than in the anterior tibial muscle at the same stage. c-ski mRNA in the $5 \mathrm{~d}$ diaphragm is also substantially lower than in the adult (Fig. 4), indicating that there is a baseline increase of $s k i$ transgene mRNA during maturation of all muscles.

At birth, the levels of c-ski transgene mRNA in future fast muscles are barely detectable (Fig. $3 a$, lane 1). Levels remain low but are more clearly detectable at 5 d (Fig. $3 a$, lane 3). By
$8 \mathrm{~d}$, there is a significant increase (Fig. $3 A$, lane 5 ) and by $12 \mathrm{~d}$, transcript levels approach the level seen in the adult (Fig. $3 a$, compare lane 7 with lanes 9 and 11). Comparison of Figures 2 and 3 shows that the increase in the levels of c-ski mRNA parallels the emergence of the hypertrophic ski phenotype.

In view of the strong correlation between the $s k i$ and IIb fiber specialization, it is significant that emergence of the hypertrophic phenotype in developing transgenic mice coincides with the transition from perinatal to IIb MHC mRNA (Wydert et al., 1987). The rise in $s k i$ transgene mRNA between 5 and 12 d implies either that maturational controls activate the MSV promoter and cause increased ski transcription or that there is a pronounced change in ski mRNA stability during this period. In either event, as shown by immunocytochemical methods 

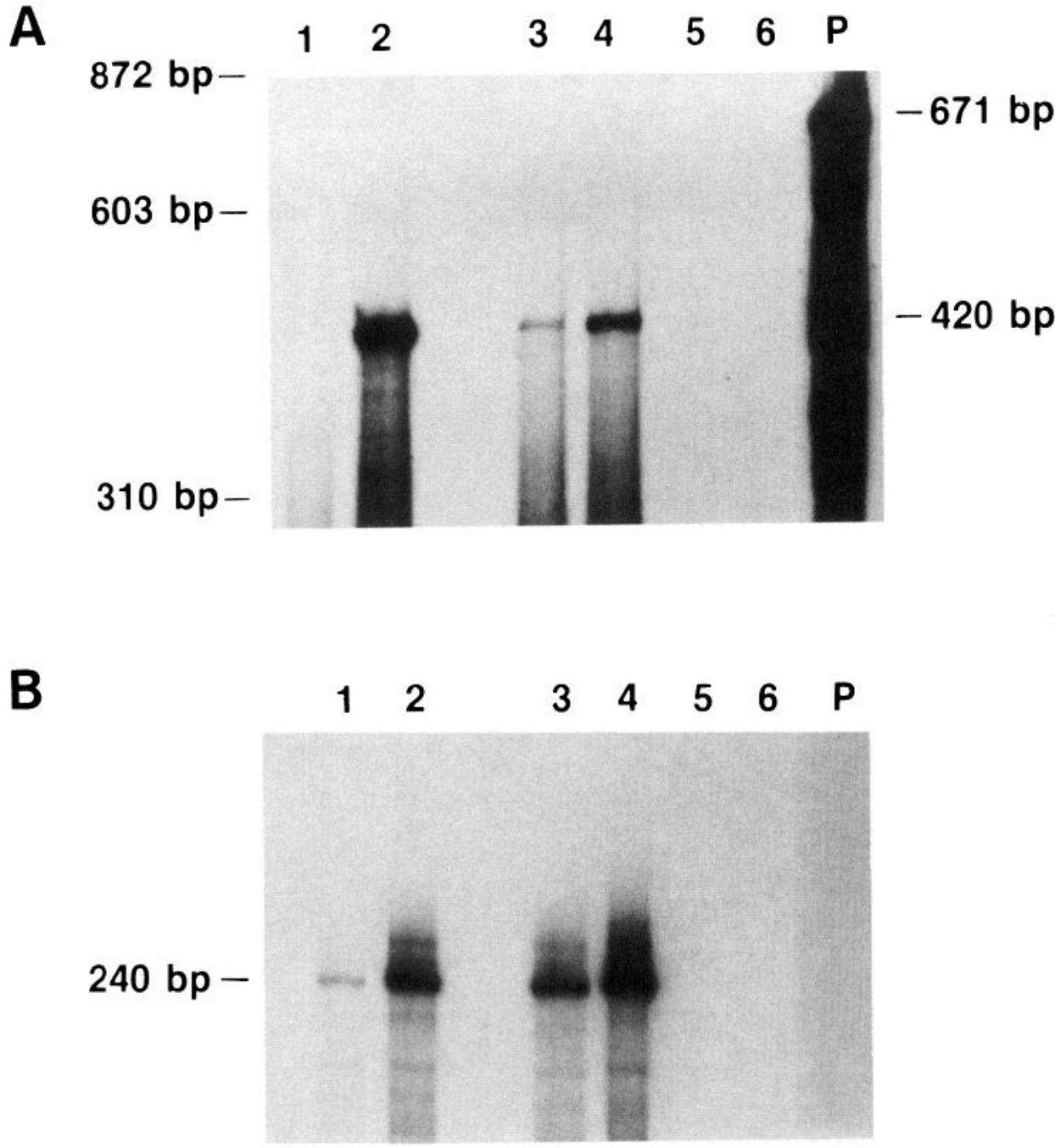

Figure 4. c-ski mRNA expression in the developing diaphragm. $A$, RNase protection assay demonstrating the differential expression of c-ski mRNA in the diaphragm and anterior tibialis muscle of transgenic mice. A 671-base uniformly labeled antisense probe (lane $P$ ), was hybridized to $15 \mu \mathrm{g}$ of total RNA. RNase-resistant fragments from hybridization with RNA from the diaphragm or anterior tibial muscles are shown in lanes 1-4. Lane 1, Leg muscle from adult normal mouse. Lane 2, Leg muscle from adult ski transgenic mouse. Lanes 3 and 4, Diaphragm from, respectively, 5-d-old and adult transgenic mice. Lane 5, tRNA control. Lane 6, Probe control. Numbers to the left are sizes in bases as indicated by a PhiX 174 Hae III digested DNA marker. Numbers to the right indicate the sizes of the probe (671) and the size of the RNA resistant fragments. The level of c-ski mRNA in the adult diaphragm approximates the level in the EDL at $8 \mathrm{~d}$ of age. $B$, RNase protection assay demonstrating the expression of endogenous $\alpha_{\mathrm{sk}}$ actin. RNase-resistant fragments from hybridizations with RNA from the anterior tibialis or diaphragm are shown in lanes 1-4 and correspond to the samples indicated in $A$. below, ski protein can accumulate in the nuclei of all of the fibers of fast muscles but growth stimulation is limited to type IIb fibers.

\section{Accumulation of $\mathrm{c}$-ski protein in developing and mature muscle}

To detect the accumulation of c-ski protein, we have used a monoclonal antibody that specifically recognizes an epitope encoded by exon 1 of ski mRNA (gift from Dr. Ed Stavnezer; see also Sutrave et al., 1990a). In the transgenic mice, c-ski protein is localized to muscle nuclei (Fig. $5 a$ ). In the mature plantaris, gastrocnemius, anterior tibial, and EDL muscles, the nuclei of all fibers, large and small, stain with the antibody (Fig. $5 b$ ) with equal intensity. This is surprising, since we have shown that in the transgenic animals, there is a correlation between fiber phenotype and fiber size (Fig. 1). In contrast to these results, nuclear staining is undetectable in the soleus and diaphragm even though these muscles contain many of the same fiber types as present in the EDL, plantaris, and other fast muscles (Fig. 5c). The distinction in staining between the adjacent muscles in the same section demonstrates the specificity of the antibody for c-ski and is in agreement with the analyses of c-ski mRNA levels in these muscles (Sutrave et al., 1990b).
Taken together, these results show that a complex system of controls regulate (1) the levels of c-ski mRNA and protein in muscle; and (2) the emergence of the hypertrophic phenotype. Muscles with low levels of c-ski mRNA and protein (soleus and diaphragm) have high functional demands and they do not hypertrophy. This would suggest that function, directly or indirectly, inhibits c-ski expression. Based on this argument, we might expect to find that there is a gradient of c-ski protein levels in fast muscles; staining with c-ski antisera following the order of recruitment of fibers. In this model, the nuclei of slow and IIa fibers should have weak staining, whereas those of IIb fibers would be intense. Within visual limits of quantitation by immunofluorescence, this does not appear to be true. However, in studies using in situ hybridization (Lana et al., in preparation) it is apparent that such a gradient does exist and that the highest levels of c-ski transcript occur in the superficial regions of muscle matching the distribution of IIb fibers.

In the developing plantaris, the earliest stage at which we were able to demonstrate nuclear staining is at $10 \mathrm{~d}$ postpartum. The increase in ski mRNA between 5 and $12 \mathrm{~d}$ is, therefore, in good agreement with the ability to identify c-ski protein in the myonuclei and with the emergence of hypertrophy. 

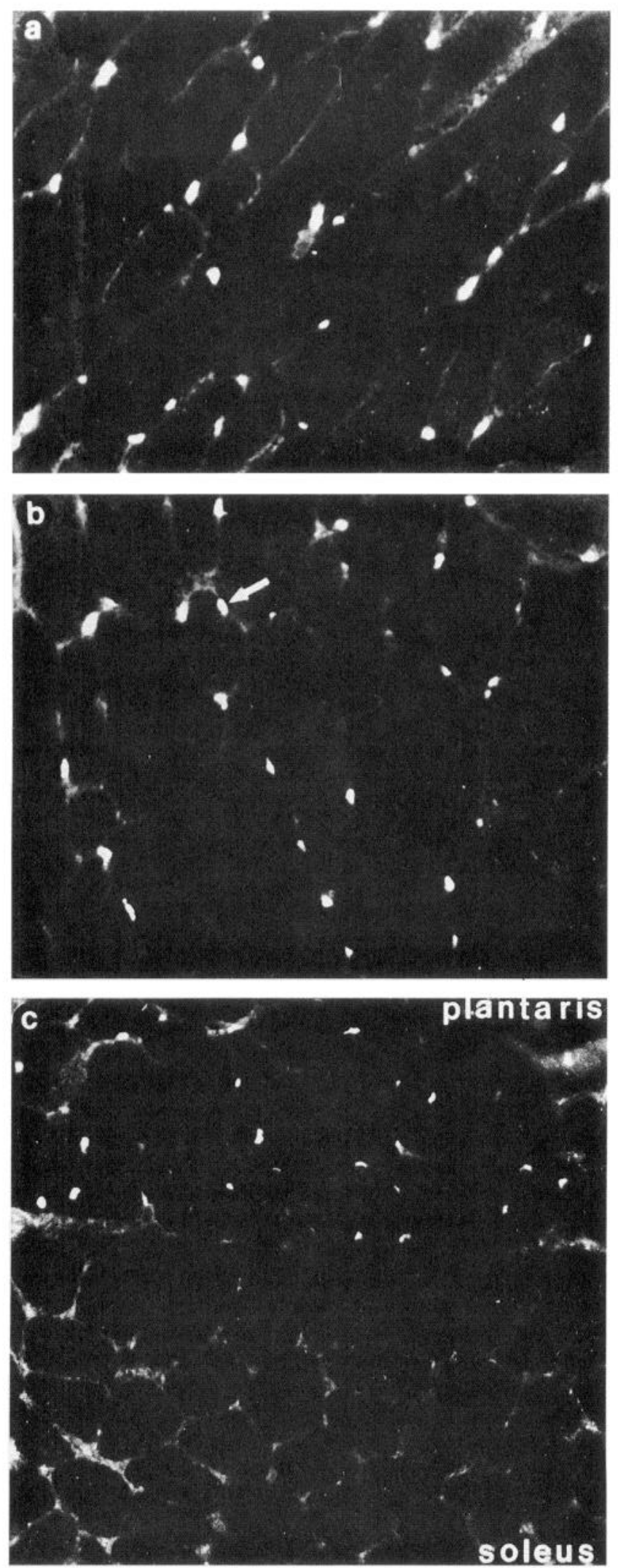

Figure 5. c-ski protein accumulation in myonuclei of transgenic mice. Oblique $(a)$ and transverse $(b$ and $c)$ sections, made through the plantaris and soleus muscles of transgenic animals, were stained with a monoclonal antibody to c-ski nuclear protein. $a$ and $b$ are from the plantaris, a fast muscle, and show that the nuclei of all fibers, large and small, stain for ski protein. Arrow in $b$ indicates a small fiber with at least one
A

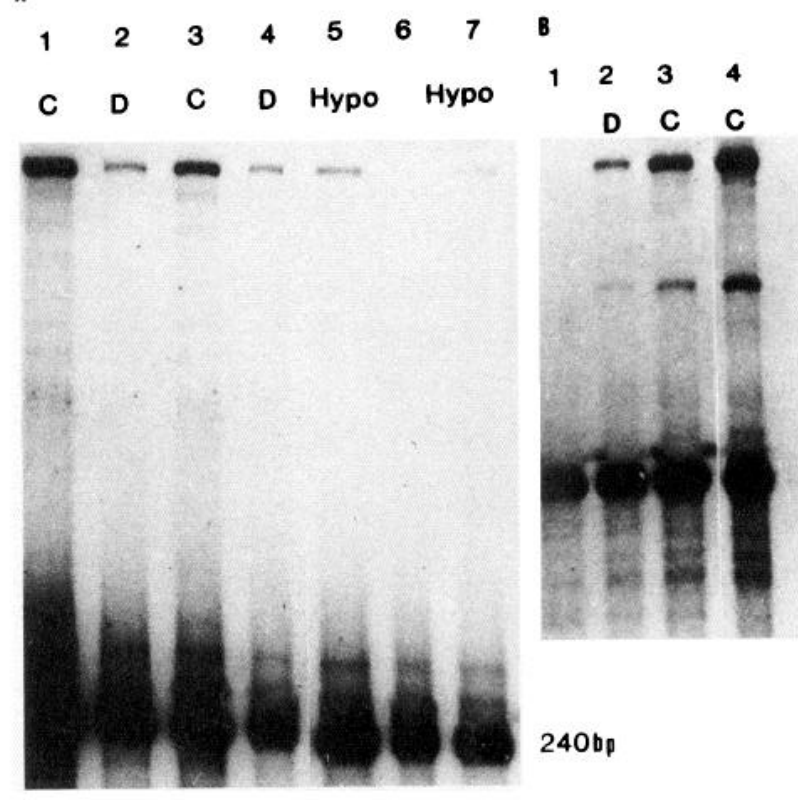

420 bp

240tp

Figure 6. RNase protection assays demonstrating the effects of denervation and of hypothyroidism on levels of c-ski mRNA in the anterior tibial muscle of transgenic mice. The assay uses a $671 \mathrm{bp}$-linked $s k i$-actin probe. $A$, Protected fragments are from lanes 1 and $3,15 \mathrm{~d}$ control c-ski anterior tibial muscle; lanes 2 and 4, contralateral $15 \mathrm{~d} \mathrm{ski}$ anterior tibial muscle after denervation at birth; lanes 5 and 6 , anterior tibial muscle from c-ski mice made hypothyroid by feeding $0.05 \%$ PTU in the drinking water from birth. Muscles were obtained after $15 \mathrm{~d}$ (lane 5) and $17 \mathrm{~d}$ (lane 7) of hypothyroidism. Compared to the control, levels of c-ski mRNA are reduced by approximately $50 \%$ in denervated and hypothyroid anterior tibial muscles. $B$, Protected fragments from the adult anterior tibial muscles of c-ski transgenic mice; lane 2, after $30 \mathrm{~d}$ denervation; lanes 3 and 4, unoperated control ski muscle. Levels of c-ski mRNA are reduced by approximately $50 \%$ after denervation.

Neural and thyroidal regulation of ski expression in developing and mature muscle

In the mouse, $T_{4}$ and $T_{3}$ values rise to maximal values during the same period that $s k i$ induced hypertrophy emerges (Leferovich and Kelly, unpublished observations). In previous studies, we and others have shown that the development of thyroid function is implicated in the maturation of fibers, specifically the transition from the expression of neonatal to IIb MHC (Gambke et al., 1983; Butler Browne et al., 1984). This switch is inhibited by hypothyroidism in both rat and mouse (Gambke et al., 1983; Russell et al., 1988). The correlation persuaded us to measure the influence of thyroid hormone on ski expression and muscle hypertrophy. Transgenic mice were made hypothyroid by feeding $0.05 \%$ PTU in the drinking water from birth. Tissues from the hypothyroid animals were analyzed at 15 and $17 \mathrm{~d}$ postpartum. Levels of ski mRNA expression in hind limb musculature of hypothyroid animals were significantly reduced

stained nucleus. Section in $c$ includes portions of the plantaris and soleus muscles. Nuclei in the plantaris are stained, whereas they are not stained in the soleus. This pattern of staining confirms the specificity of the $s k i$ antibody. The antibody stains interstitial connective tissue nonspecifically. Magnification $\times 200$. 
22 DAY

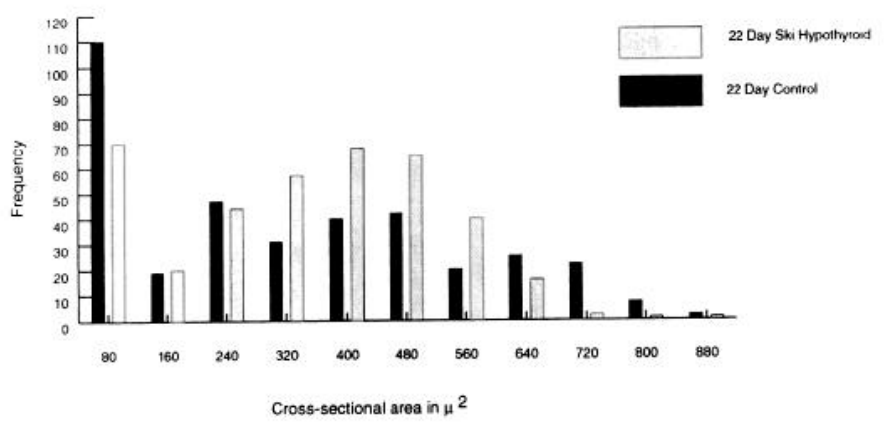

Figure 7. Effects of hypothyroidism on fiber size in developing EDL of c-ski transgenic mice. Cross-sectional areas of fibers from 22-d-old control mice and from 22-d transgenic mice made hypothyroid by supplying $0.05 \%$ PTU in the drinking water from birth. There is no hypertrophy in the muscles of hypothyroid transgenic mice.

compared to control levels (Fig. 6). The reduced levels of c-ski message apparently cause ski protein levels to fall below a critical threshold for there was no hypertrophy of muscles in the hypothyroid transgenic mice (Fig. 7).

The effect of thyroid hormone on the expression of the c-ski transgene can be explained as a direct effect on the transcriptional activity of the MSV LTR, since this LTR is known to contain a thyroid response element and to respond positively to increased levels of thyroid hormone (Sap et al., 1989). The alternative explanation, which we prefer, is that IIb fiber differentiation is inhibited in hypothyroid animals; in these circumstances, the "permissive" environment for activation of the MSV promoter and increased c-ski transgene expression is unavailable.

To examine the influence of innervation on the regulation of ski expression in developing transgenic mice, we have transected the sciatic nerve in the proximal, left hind limb of three transgenic mice at birth. Under these circumstances, IIb fiber differentiation is known to be compromised (Lowrie et al., 1982, 1990). Levels of $s k i$ transcript in the innervated and contralateral, denervated EDL were compared at $15 \mathrm{~d}$ of age. Denervated limbs were atrophic and levels of ski mRNA expression were reduced by approximately $50 \%$ compared to the innervated cohort (Fig. 6A). We have also studied the expression of c-ski in the adult anterior tibial muscle after denervation. Thirty days after unilateral denervation, the left anterior tibial muscle was severely atrophic and the levels of c-ski expression were reduced by $50 \%$ compared to controls (Fig. $6 \mathrm{~B}$ ).

Taken together, the neurectomy studies show that high levels of c-ski mRNA expression require continual neural stimulation; but whether this is a direct or indirect effect is unknown. If the effect is direct, it would require that in our transgenic mice, the viral MSV LTR is curiously responsive to the nerve. This would be a surprising property of the MSV promoter. Alternatively, neural regulation may be indirect, mediated primary on IIb fiber differentiation and secondarily upon levels of c-ski expression. This explanation is consistent with our analysis of thyroidal effects on ski expression and can be correlated with the studies on development of c-ski expression and IIb fiber hypertrophy. The results, however, shed no light on the properties within the environment of IIb fibers that may lead to such remarkably specific amplification of c-ski expression and fiber hypertrophy.

\section{References}

Buller AJ, Eccles JC, Eccles RM (1960) Differentiation of fast and slow muscles in the cat hind limb. J Physiol (Lond) 150:399-416.

Butler Brown AE, Herlicoviez D, Whalen RG (1984) Effects of hypothyroidism on myosin isoenzyme transitions in developing rat muscle. FEBS Lett 166:71-75.

Colmenares C, Stavnezer E (1989) The ski oncogene induces muscle differentiation in quail embryo cells. Cell 59:293-303.

Colmenares C, Sutrave P, Hughes SH, Stavnezer E (1991) Activation of the c-ski oncogene by over-expression. J Virol 65:4929-4935.

DiMario JX, Uzman A, Strohman RC (1991) Fiber regeneration is not persistent in dystrophic (MDX) mouse skeletal muscle. Dev Biol 148:314-321.

Gambke B, Lyons G, Haselgrove J, Kelly AM, Rubinstein N (1983) Thyroidal and neural control of myosin transitions during development of rat fast and slow muscles. FEBS Lett 156:335-339.

Khillan JS, Oskarsson MK, Propst F, Kuwabara T, Vande G, Woude F, Westphal H (1987) Defects in lens fiber differentiation are linked to c-mos overexpression in transgenic mice. Genes Dev 1:1327-1335.

Leader DP, Gall I, Campbell P, Frischauf AM (1986) solation and characterization of cDNA clones from mouse skeletal muscle actin mRNA. DNA 5:235-238.

Li Y, Turck CM, Teumer JJ, Stavnezer E (1986) Unique sequence, $s k i$, in Sloan-Kettering avian retrovirus with properties of a new cell derived oncogene. J Virol 57:1065-1072.

Lowrie MB, Krishnan S, Vrbova G (1982) Recovery of slow and fast muscles following nerve injury during early post-natal development in the rat. J Physiol (Lond) 331:51-66.

Lowrie MB, Shahani U, Vrbova G (1990) Impairment of developing fast muscle after nerve injury in the rat depends upon the period of denervation. J Neurol Sci 99:249-258.

Lyons GE, Ontell M, Cox R, Sassoon D, Ott M-O, Buckingham M (1990) The expression of myosin genes in developing skeletal muscle in the mouse embryo. J Cell Biol 111:1465-1476.

Narusawa M, Fitzsimons RB, Izumo S, Nadal-Ginard B, Rubinstein NA, Kelly AM (1987) Slow myosin in developing rat skeletal muscle. J Cell Biol 104:447-459.

Russell SD, Cambon N, Nadal-Ginard B, Whalen RG (1988) Thyroid hormone induces a nerve-independent precocious expression of fast myosin heavy chain mRNA in rat hind limb skeletal muscle. J Biol Chem 263:6370-6374.

Sap J, Munoz A, Schmitt J, Stunnenberg H, Vennstrom B (1989) Repression mediated as a thyroid response by the v-erb-A oncogene product. Nature 341:242-244.

Schiaffino S, Gorza L, Sartore S, Saggin L, Ausoni S, Vianello M, Gunderson K, Lomo T (1989) Three myosin heavy chain isoforms in type II skeletal muscle fibers. J Muscle Res Cell Motil 10:197-205.

Stavnezer E (1988) The ski gene. In: The oncogene handbook (Reddy EP, Skalka A, Curran T, eds), pp 393-401. Amsterdam: Elsevier.

Stavnezer E, Gerhard DS, Binari RD, Balzas I (1981) Generation of transforming viruses in culture of chicken fibroblasts infected with avian leukosis virus. J Virol 39:920-934.

Stavnezer E, Barkas AE, Brennan LA, Brouder D, Li Y (1986) Transforming Sloan-Kettering viruses generated from the cloned $v$-ski oncogene by in vitro and in vivo recombination. J Virol 57:1073-1083.

Sutrave P, Hughes S (1989) Isolation and characterization of three distinct cDNAs for the chicken c-ski gene. Mol Cell Biol 9:40464051 .

Sutrave P, Hughes SH (1991) The ski oncogene. Oncogene 6:353356.

Sutrave P, Copeland TD, Showalter SD, Hughes SM (1990a) Characterization of chicken c-ski oncogene products expressed by retrovirus vectors. Mol Cell Biol 10:3137-3144

Sutrave P, Kelly AM, Hughes SH (1990b) Ski can cause selective growth of skeletal muscle in transgenic mice. Genes Dev 4:14621472 .

Sutrave P, Leferovich J, Kelly AM, Hughes SH (1992) c-ski expression can increase the skeletal musculature of transgenic mice. In: Raven Press series on molecular and cellular biology, Vol 2, Neuromuscular development and disease (Kelly AM, Blau HM, eds), pp 107-114. New York: Raven.

Wydert A, Barton P, Harris AJ, Pinset C, Buckingham M (1987) Developmental pattern of mouse skeletal myosin heavy chain gene transcripts in vivo and in vitro. Cell 49:121-129. 